\title{
Bassey Edem Antia, Indeterminacy in Terminology and LSP: Studies in Honour of Heribert Picht
}

Amsterdam, Philadelphie : John Benjamins, 2007

\section{John Humbley}

\section{(2) OpenEdition}

\section{Journals}

Édition électronique

URL : http://journals.openedition.org/asp/306

DOI : $10.4000 /$ asp.306

ISBN : 978-2-8218-0404-3

ISSN : 2108-6354

\section{Éditeur}

Groupe d'étude et de recherche en anglais de spécialité

\section{Édition imprimée}

Date de publication : 1 décembre 2007

Pagination : 189-193

ISSN : 1246-8185

\section{Référence électronique}

John Humbley, «Bassey Edem Antia, Indeterminacy in Terminology and LSP: Studies in Honour of

Heribert Picht », ASp [En ligne], 51-52 | 2007, mis en ligne le 31 décembre 2009, consulté le 22 mars 2021. URL : http://journals.openedition.org/asp/306 ; DOI : https://doi.org/10.4000/asp.306

Ce document a été généré automatiquement le 22 mars 2021.

Tous droits réservés 


\section{Bassey Edem Antia, Indeterminacy in Terminology and LSP: Studies in Honour of Heribert Picht}

Amsterdam, Philadelphie : John Benjamins, 2007

John Humbley

\section{RÉFÉRENCE}

Antia, Bassey Edem. 2007. Indeterminacy in Terminology and LSP: Studies in Honour of Heribert Picht. Amsterdam, Philadelphie : John Benjamins. Collection Terminology and Lexicography Research and Practice. Volume 8. 233 p. ISBN 978-902722-332-6. 
1 En abordant le thème de l'indétermination, traduction approximative de "indeterminacy» de ce nouveau recueil, Bassey Edem Antia ouvre les études de terminologie et plus généralement de langues de spécialité (LSP) à plusieurs autres disciplines voisines. En s'ouvrant à d'autres approches, les études de langues de spécialité se remettent ainsi en question et renouvellent très utilement leur problématique.

2 Depuis longtemps les chercheurs s'efforçaient de démontrer comment la communication spécialisée réalise la précision et élimine l'ambiguïté. Cependant, plus récemment, d'une part dans la pratique des LSP juridiques, mais aussi au niveau théorique, le phénomène

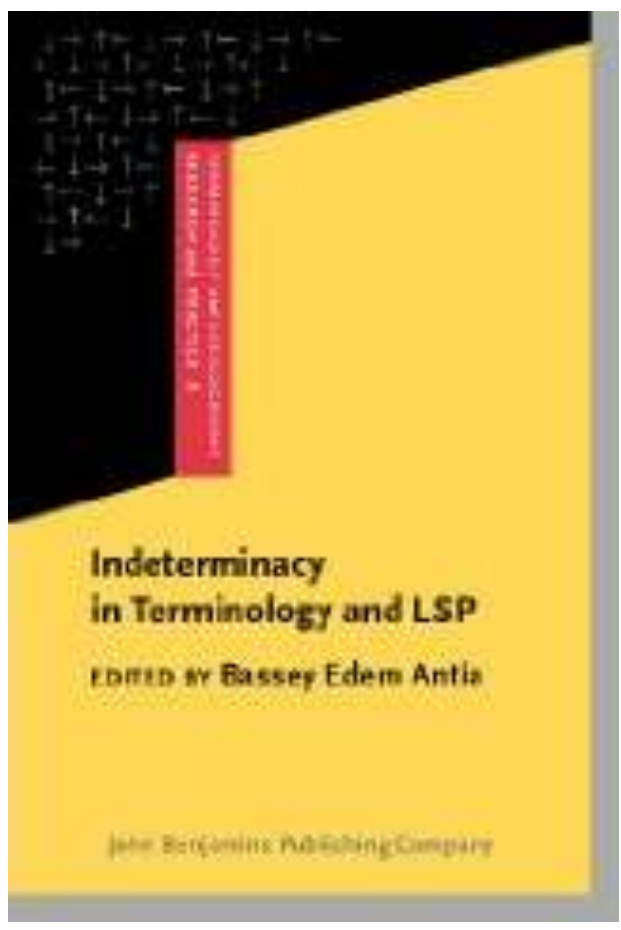
inverse, le caractère vague ou imprécis des systèmes et des discours de spécialité commence à faire l'objet d'une étude spécifique. Par ce biais, la terminologie en particulier reprend le dialogue avec d'autres courants de la linguistique, en s'attachant à dénouer toute la complexité de son sujet d'étude. Cet ouvrage peut donc être utile aux chercheurs en anglais de spécialité dans la mesure où il ouvre de nouvelles perspectives dans un esprit pluridisciplinaire, ainsi que par les thèmes évoqués, qui rejoignent souvent ceux de la revue ASp : le langage juridique, le rôle de la métaphore en langue de spécialité (économique surtout) et l'analyse textuelle de la variation synonymique. Malgré les origines diverses des auteurs, qui appartiennent à onze nationalités différentes pour quatorze chapitres, l'approche qui prédomine est celle de l'Europe du Nord, offrant ainsi une initiation commode à ces courants de pensée, puisque tous les articles sauf un sont rédigés en anglais.

3 La préface de B. E. Antia situe la problématique par rapport à la production scientifique de Heribert Picht, à qui ce recueil est dédié. Parfois présenté en France comme un partisan de la terminologie normative, H. Picht n'a cessé d'innover sur le plan théorique: tout en se réclamant de la priorité du concept en terminologie, il a largement contribué à élargir le sujet de la recherche. Il est parmi les premiers à montrer la pertinence des verbes et des adjectifs pour la terminologie, de la phraséologie et des aspects communicatifs. Il prône depuis longtemps la prise en compte de la terminologie en intelligence artificielle. Le chapitre 14, le dernier du recueil, est en fait une très riche " bibliovita », ou bibliographie analytique préparée par Caroline Popp. Les quatre grands thèmes présentés dans le recueil reprennent ainsi les principales préoccupations scientifiques de H. Picht, à savoir des perspectives lexicale, épistémologique et ontologique ${ }^{1}$. Cette recension porte essentiellement sur les deux premières.

4 Øvin Anderson (chapitre 1), pionnier de la réflexion sur l'indétermination en langues de spécialité, ouvre la discussion en proposant une analyse de cet aspect en linguistique 
théorique. Il montre d'une part que les linguistes l'incorporent de différentes façons dans leur théorie; d'autre part il fait une démonstration des degrés d'indétermination qu'il convient d'analyser en les factorisant. Les exemples sont indiqués en norvégien, mais la traduction anglaise fournit tous les éléments nécessaires à la compréhension. Cet article constitue ainsi un lien entre la linguistique théorique ("mainstream", comme l'écrit l'auteur) et l'étude des langues de spécialité.

Margaret Rogers (chapitre 2) aborde la question de l'indétermination par le biais de la variation terminologique telle qu'elle est représentée dans un texte hautement technique aussi bien au niveau de l'original que dans les deux versions traduites, qui constituent le corpus soumis à l'analyse. On aurait pu s'attendre à ce que les termes qui désignent des objets matériels surspécifiés soient toujours représentés par la même dénomination, d'autant plus que le contexte est celui des appareils médicaux, pour lesquels la sécurité représente un enjeu particulièrement important. Or, il s'avère que le rédacteur du texte original, comme les deux traducteurs, préfère dans certains cas des variations de différents ordres : réductions de termes (" clippings ») hyperonymes, synonymes, sans que chaque occurrence puisse faire l'objet d'une explication simple. M. Rogers montre que les théories linguistiques (par exemple celle de W.V. Quine (1966) en matière de représentations linguistiques ou encyclopédiques) doivent être revues à la lumière des jeux de référence à l'œuvre dans un texte et dans ses différentes traductions. Bref, c'est une étude innovante sur l'explication de la synonymie terminologique en traduction par rapport à la rédaction et un exemple d'analyse textuelle dans un recueil plutôt « orienté système ».

6 Sergey Griniewicz (chapitre 3), mieux connu dans le milieu des terminologues sous le nom de Grinev, adopte pour sa part l'anthropogénèse comme cadre d'analyse de la détermination du langage humain. Il s'efforce de démontrer que l'évolution à partir d'un état de langue (celui du vieil anglais et du vieux slavon dans l'article) caractérisé par une très forte indétermination sémantique va vers une précision croissante au fur et à mesure des progrès scientifiques. Dans cette perspective, la préoccupation des concepts flous de certaines sciences modernes ne relève pas d'un retour en arrière mais d'une nouvelle quête d'adéquation entre la pensée scientifique et les moyens - surtout linguistiques - d'expression.

7 Klaus-Dirk Schmitz (chapitre 4) rappelle que l'indétermination reste toujours un handicap dans de nombreux secteurs, dont celui de la localisation, et il énumère les formes qu'elle peut prendre. Lorsqu'on localise un logiciel, il est indispensable d'adopter une terminologie cohérente et systématique, non seulement à l'intérieur de la documentation propre au logiciel, mais aussi dans celle des autres applications avec lesquelles le logiciel en question est susceptible de fonctionner. K.-D. Schmitz met en garde contre une forme d'indétermination d'origine culturelle qui s'exprime à la fois par la langue et par les icônes. Les tournures souvent métaphoriques tirées de la culture américaine en général ou de celle des «nerds» en particulier («opération illégale ", "erreur fatale », etc.), tout comme les icônes qui sont soit culturelles (la corbeille représentée par une poubelle new-yorkaise) soit linguistiques (le tableur représenté par une table) sont autant de pièges qu'il convient de neutraliser lors de la phase précédente : l'internationalisation.

8 Gerhard Budin (chapitre 5), pour sa part, s'efforce de placer l'indétermination terminologique dans son cadre épistémologique. En effet, l'indétermination caractérise non seulement les sciences récentes comme la physique quantique mais surtout le post- 
modernisme en général sous toutes ses formes. G. Budin considère que la linguistique textuelle telle qu'elle est développée par R. de Beaugrande en particulier, qui privilégie une démarche caractérisée par une série d'oppositions actualisées de façon conjoncturelle, constitue une manifestation intéressante de ce courant de pensée.

Une façon radicale de supprimer l'indétermination est d'établir des dichotomies rigides, pratique qui caractérise l'émergence de la terminologie en tant que discipline : on oppose ainsi mot à terme, sémasiologie à onomasiologie, etc. Johan Myking, qui estime que ces distinctions nettes représentent une simplification abusive, étudie les différentes façons dont ces dichotomies ont été exploitées non seulement pour en quelque sorte marquer le terrain, mais aussi pour négocier des ententes générales ou partielles au sujet des objectifs et des méthodes de la discipline. Dans ce sixième chapitre, l'auteur renonce à analyser une des dichotomies les plus souvent évoquées en terminologie, celle de l'opposition description/prescription, mais c'est pour donner la parole à $\mathrm{H}$. Picht, qui, lors d'une conférence récente prononcée en Russie, la transforme en une dialectique dynamique, comme R. de Beaugrande le préconise. Cet article est particulièrement intéressant pour ceux qui étudient l'évolution de la terminologie comme composante de la linguistique.

Une des qualités de ce recueil est l'ouverture aux traditions de recherche injustement méconnues; on apprécie particulièrement de ce point de vue l'analyse que font $\mathrm{V}$. Leitschek et S. Shelov (chapitre 7), qui puisent dans les sources russes différents types de définition. Ils reconnaissent que la définition complètement non ambiguë n'est pas toujours possible, et ils analysent les diverses formes que peuvent prendre les définitions « floues ». Clairement, il s'agit ici d'un article de fond pour le terminologue.

Birthe Toft (chapitre 8) aborde un sujet souvent évoqué par les chercheurs en anglais de spécialité : le rôle de la métaphore en théorie économique. Elle analyse l'origine du concept de l'équilibre en macro-économie, qu'elle met en relation avec la physique de Newton, mais aussi, de nos jours, avec la théorie expérientialiste de Lakoff et Johnson, en passant par celles des terminologues (E. Wüster bien sûr, mais aussi F. Riggs), des philosophes (Adolphsen en particulier), des économistes (Robinson, Maclup, McCloskey) dont elle examine les rapports souvent inattendus.

Le rôle du "vague " dans les textes normatifs a fait l'objet d'un recueil désormais célèbre (Bahtia 2005). Ingrid Simonæs (chapitre 9) poursuit la réflexion dans le domaine juridique. Elle examine en particulier les relations entre le concept en droit et en terminologie, sous l'angle des définitions en compréhension et en extension. Une des différences qu'elle perçoit entre les deux est l'impossibilité en droit de donner une définition en extension de façon exhaustive; c'est donc aux juges et au système juridique de déterminer si tel ou tel cas relève d'une définition volontairement générale (ou vague). Elle explore également les implications proprement linguistiques de la question, en faisant appel en particulier aux linguistes allemands Abraham et Pinkal, tout en s'interrogeant sur la distinction wustérienne entre sens et concept. Les exemples relèvent du droit allemand, mais les concepts qu'elle présente ("good faith", " for good cause »), soulèvent les mêmes problèmes dans d'autres systèmes juridiques.

13 Dans le seul article (chapitre 10) qui ne soit pas en anglais, ${ }^{2}$ Reiner Arntz et Peter Sandrini situent le problème de la dichotomie entre le vague et le précis dans le cadre du langage juridique international, impliquant une démarche à la fois juridique et linguistique. Ils soulignent l'importance de l'histoire pour bien comprendre les différences qui existent entre les concepts juridiques de différents systèmes. Ils 
énoncent, dans un développement particulièrement utile, les étapes de la recherche en terminologie juridique, en soulignant les spécificités par rapport aux principes de la terminologie générale. Les exemples sont tirés de la paire de langues allemand/italien, mais ils prennent aussi en compte le système juridique de l'Union européenne. Malgré l'absence de l'anglais au niveau du corpus étudié, les principes évoqués sont très utiles aux anglicistes travaillant dans le domaine juridique comparé.

Sue Ellen Wright (chapitre 11), connue pour ses travaux dans le domaine de la normalisation, situe sa réflexion dans le paradoxe apparent des ambitions de l'ingénierie de la connaissance, qui recherchent la structure et la précision à un moment où les linguistes et les philosophes rejettent le structuralisme et l'idée de la détermination. Tout en se plaçant du côté des premiers, elle tente de réconcilier les deux approches en proposant une présentation globale des ressources de la représentation de la connaissance (Knowledge Representation Resources), accessible par ailleurs à son site ${ }^{3}$. Enfin, dans le dernier volet, Bodil Nistrup Madsen (chapitre 12) présente les principes de la construction d'ontologie dans une perspective d'ingéniérerie de la connaissance et de traduction, et elle montre comment on tient compte de l'indétermination tout en veillant à ce que les représentations soient complètement non ambiguës. Anita Nuopponen (chapitre 3) aborde, elle aussi, la question de la modélisation de la connaissance, mais en prenant comme exemple la cérémonie du thé au Japon. Les critères qu'elle dégage et leur agencement seront utiles à ceux qui s'intéressent à la terminologie systématique.

Il est vrai que ce recueil aborde les problèmes de l'indétermination dans les langues de spécialité d'un point de vue surtout terminologique. Cependant l'ouverture vers d'autres disciplines et la prise en compte explicite de la linguistique théorique, sans oublier la présentation des résultats des recherches importantes menées dans les pays de langues germaniques, enrichiront le débat sur les perspectives de recherche dans le domaine des langues de spécialité.

\section{BIBLIOGRAPHIE}

Bhatia, V. J. et al. 2005. Vagueness in Normative Texts. Studies in Language and Communication, vol. 23. Berne : Peter Lang Verlag : Quine, W.V. 1966.

« Meaning and translation », On Translation, R.A. Brower (dir.). 148-172. New York : Oxford University Press.

\section{NOTES}

1. Modélisation des connaissances.

2. Il est en allemand.

3. <http://appling.kent.edu/ResourcePages/LTStandards/Chart/kos.html>. 


\section{AUTEURS}

\section{JOHN HUMBLEY}

Université Paris Diderot 